\title{
COMENTARIOS SOBRE LA INSULARIDAD DE LA GRACIOSA EN EL NUEVO ESTATUTO DE AUTONOMÍA DE CANARIAS
}

\author{
Noel Armas Castilla \\ Universidad de Sevilla
}

\section{RESUMEN}

El nuevo Estatuto de Autonomía de Canarias ha introducido una serie de cuestiones de gran interés para el Derecho administrativo. El objeto en el que se centra este artículo es la consideración estatutaria de La Graciosa como una «isla» contra la anterior denominación de «islote». Este nuevo estatus debe ser estudiado para conocer cómo el marco organizativo de La Graciosa encaja en un contexto tan particular como el de las islas Canarias. Ello nos lleva a analizar el sistema de representación y gobierno de este enclave para equiparar, o al menos asimilar, su nuevo estatus con la transferencia de autonomía subjetiva, objetiva e instrumental que debería asistirle. La autonomía local de La Graciosa habría de materializarse con la institucionalización de las correspondientes Administraciones de gobierno local o con la reorganización de sus relaciones con el resto de niveles administrativos que le son propios como el Cabildo de la vecina isla de Lanzarote, a la que pertenece administrativamente, el Gobierno de Canarias y el del Estado.

Palabras clave: autonomía, isla, comunidad autónoma, Canarias, pedanía, gobernanza, participación, organización territorial, Estatuto de Autonomía, Cabildo Insular.

\section{COMMENTS ON THE INSULARITY OF LA GRACIOSA IN THE NEW STATUTE OF AUTONOMY OF THE CANARY ISLANDS}

\section{Abstract}

The new Statute of Autonomy of the Canary Islands has introduced a serie of interesting features acording to administrative law. The feature that this article focuses is the legal consideration of La Graciosa as an "island." This new status must be studied in order to understand how the organisational framework of La Graciosa fits in a territorial context as peculiar as the Canary Islands. This requires a study of the system of representation and government of this island in order to equate its new status with the transfer of the appropriate autonomy which should assist it. This autonomy should be materialised in the institutionalisation of the corresponding local government administrations or the reorganisation of its relations with its other administrative levels as the Council of the neighbouring Island of Lanzarote, the Canary Islands Government, and that of the State.

KEYwORDs: autonomy, island, Canary Islands, district, governance, participation, territorial organisation, Statute of Autonomy, Island Council. 


\section{INTRODUCCIÓN}

La aprobación de la Ley 1/2018, de 5 de noviembre, de reforma del Estatuto de Autonomía de Canarias trajo consigo la satisfacción de algunas demandas que determinados sectores de la vida política y social del archipiélago habían venido formulando desde hace un tiempo. No en vano, el caso canario era uno de los pocos en los que aún no se había practicado la renovación de su primer Estatuto de Autonomía, debido, entre otras cosas, a la dilación que los tiempos parlamentarios y negociaciones políticas habían producido en un marco tan poco propicio como han sido estos últimos ejercicios de legislatura precipitadamente cerrados.

Son bastantes las novedades que introdujo el nuevo Estatuto, y de entre ellas, una de las más interesantes podría situarse en la reforma del régimen electoral y de representatividad parlamentaria con que algunos sectores sociales y políticos reivindicaban la necesidad de abandonar el sistema de triple paridad entre las dos provincias, entre las denominadas islas mayores y entre las hasta entonces cinco islas menos pobladas con las dos más pobladas. En el bronco debate de redacción de la propuesta de Estatuto, desarrollado en el seno del parlamento autonómico, se fraguó el acuerdo de aprobar un sistema mixto que tuviera en cuenta por un lado la representatividad de cada isla en la Cámara regional y por otro lado la reserva de un determinado número de escaños a una lista regional que aproximase la composición de la Cámara al principio de igualdad de valor de los votos emitidos por el conjunto de electores canarios, frente al anterior sistema electoral, que, si bien había sido autorizado por la STC 225/1998, de 25 de noviembre ${ }^{1}$, había dado origen a una importante diferencia de valor entre los votos de los electores de una isla y otra.

No obstante, y por no perdernos en el interesante océano jurídico que ha abierto al análisis este nuevo Estatuto, nos interesa centrarnos en una de las novedades de interés para la rama del Derecho administrativo como es la nueva enumeración de las islas Canarias, en la que pasa a contabilizarse a la (ahora) isla de La Graciosa; y que se concreta en el art. 65.1 del nuevo Estatuto, que establece que «la organización territorial de Canarias se integra por las islas de El Hierro, Fuerteventura, Gran Canaria, La Gomera, Lanzarote, La Palma y Tenerife. La isla de La Graciosa estará agregada administrativamente a Lanzarote, así como los islotes de Alegranza, Montaña Clara, Roque del Este y Roque del Oeste y el de Lobos a Fuerteventura.» El objetivo pues, de este artículo es el de determinar la traducción de esta proclamación en el campo del Derecho administrativo, y contribuir a la apertura de un debate jurídico en el que se resuelva la situación organizativa a la que la isla de La Graciosa se puede asimilar.

${ }^{1}$ STC 225/1998, de 25 de noviembre (BOE núm. 312, de 30 de diciembre de 1998). 


\section{2. ¿QUÉ CONSECUENCIAS JURÍDICAS COMPORTA DICHA PROCLAMACIÓN?}

Sin lugar a dudas, desde un punto de vista administrativo, el signo más reconocible de la isla en el archipiélago canario es el de su gobierno y administración al abrigo de su correspondiente Cabildo Insular, entidades reconocidas y reguladas en los arts. 65 y siguientes del nuevo Estatuto, así como en la legislación específica protagonizada por la Ley 8/2015, de 1 de abril, de Cabildos Insulares. Este tipo de entidades, desde la creación de su versión moderna en el año 1912, y a lo largo de sus diferentes reformas normativas, ha venido experimentando un crecimiento tal que ha desplazado a la figura de las diputaciones provinciales en las islas, vaciando primero y sustituyendo después a estos institutos en el ámbito territorial de cada una de las islas a excepción de La Graciosa, que en todo momento ha formado parte de la organización insular de Lanzarote y municipal de Teguise.

Precisamente, el caso de La Graciosa es el más particular desde el prisma del Derecho administrativo, porque de su realidad demográfica y económica se desprende una dificultad para su organización y administración que requeriría de fórmulas ad hoc para su superación. Nos referimos en este punto al hecho de que la figura del Cabildo como tal está llamada a la organización y coordinación de los municipios de su ámbito territorial, así como a la facilitación de relaciones entre la isla y el Gobierno de Canarias, de modo que sirven a una naturaleza bifronte de organización de la realidad interna de la isla y a su relación con el Ejecutivo regional, como corresponde a un ámbito de insularidad ( $\mathrm{y}$ a veces doble insularidad) que condiciona el desarrollo general de cada isla en Canarias.

En un ejercicio de aplicación de esta figura tradicional para la hipotética constitución de una administración en La Graciosa, contemplamos la concurrencia de diversos problemas en la isla, en la que entre otras circunstancias se da el hecho de que su población se reparte en dos núcleos poblacionales: Caleta de Sebo (730 habitantes) y Pedro Barba (3 habitantes), de acuerdo con los datos ofrecidos por el INE para el año 2018. Este dato poblacional, unido al hecho de que el asentamiento permanente de personas es una realidad históricamente reciente, ha propiciado que estas poblaciones hayan estado circunscritas hasta la actualidad al municipio de Teguise, en la isla vecina de Lanzarote. El hecho de que La Graciosa se haya administrado como enclave integrado en el municipio de Teguise, que en su traducción organizativa se ha venido saldando con la asignación de un concejal específico para la atención y gobierno de La Graciosa, supone el reconocimiento de una realidad geográfica y social específica en este enclave; sin embargo, esta forma de gestión es susceptible de revisión, especialmente si considerásemos que la proclamación de la insularidad de La Graciosa requiere que se la dote de contenido, con lo que tal vez la actual organización de esta isla sea una solución que no pueda tener una trayectoria mucho más duradera en el tiempo.

Fuera como fuese, nos llama poderosamente la atención el hecho de que no se haya podido encontrar hasta la fecha una solución jurídica que equipare a La Graciosa no ya con el resto de islas del archipiélago canario, sino con el resto de muni- 
cipios, cuestión que puede ser ahora más interesante de abordar habida cuenta de la nueva consideración insular de este territorio.

Claro que esta cuestión no es baladí, menos en los tiempos que corren, donde las sucesivas reformas sobre el régimen local han ido limitando la posibilidad de creación de nuevos municipios mediante la obligatoriedad de que los municipios resultantes de una escisión de un territorio para la creación de un municipio independiente está condicionada a que el municipio resultante cuente al menos con 5000 habitantes, a que los municipios transformados sean financieramente sostenibles, a que cuenten con recursos suficientes para el cumplimiento de las competencias municipales y a que ello no suponga disminución en la calidad de los servicios que venían siendo prestados, tal y como establece el art. 13 de la ley 7/1985, de 2 de abril, reguladora de las bases del régimen local, cuya redacción además prefiere sugerir una tendencia hacia la fusión de municipios antes que hacia su segregación.

En todo caso, este punto de debate debe contemplar algunos aspectos muy específicos que influirían en la sostenibilidad financiera del municipio de Teguise y, especialmente, de un hipotético municipio de La Graciosa, como son el hecho de que determinados servicios mínimos, de los contemplados en el art. 26 de la Ley $7 / 1985$, tendrían un ahorro considerable atendida la inexistencia de carreteras asfaltadas en el interior del enclave y que el único medio de acceso a la isla (el marítimo) no sería responsabilidad de Ayuntamiento alguno; y, sin embargo, sí deberían asumir algunas competencias de ejecución costosa a la vista de las características presentes en la realidad de la isla como son las relacionadas con la gestión del medio ambiente o el turismo, que presentan unos valores muy importantes para el ámbito de La Graciosa. Sin embargo, todo este análisis, que debería ser más profundo y riguroso, quedaría en papel mojado atendiendo al hecho (ya anteriormente apuntado) de que el enclave de La Graciosa está habitado por aproximadamente 733 personas, incumpliendo objetivamente uno de los requisitos exigidos por la legislación de régimen local como son el número mínimo de habitantes para su segregación.

Precisamente, y en atención a las reformas en materia de régimen local que pueden traerse a colación para la búsqueda de una alternativa para su organización autónoma, no podemos olvidar que una hipotética consolidación de La Graciosa como pedanía de Teguise no vendría a aportar nada en el plano competencial ni de gestión, dada la negación de personalidad jurídica a estos entes operada por la ley 27/2013, de 27 de diciembre, de racionalización y sostenibilidad de la Administración Local. Además, no está claro que los habitantes de esta isla deseen una forma concreta de autonomía, lo que debería resolverse en un plano más participativo en el seno de la sociedad graciosera.

Con todo ello, el foco de este artículo no pretende bendecir ninguna solución concreta: primero, porque no pretende esta investigación jurídica suplantar el campo de actuación de la decisión política, sino observar la situación de esta isla y su posición en el tablero de la organización jurídico-territorial, y segundo, porque no queda clara cuál es la voluntad del conjunto de vecinos de La Graciosa con respecto a su futuro administrativo, con lo cual no se trata de introducir a la fuerza la puesta en marcha de una administración sobredimensionada dadas las caracterís- 
ticas demográficas de la isla, sino, muy al contrario, de proponer y ejecutar un sistema de organización, administración, representación y participación ad hoc que, precisamente, sea sensible con la realidad de insularidad extrema y sus especiales circunstancias económicas, sociales, medioambientales y políticas de su particular hecho insular.

En este sentido, es evidente pues que la actual coyuntura económica hace desaconsejable, y el ordenamiento vigente hace imposible, la creación de una organización administrativa local propia y autosuficiente que pretenda hacer de este enclave una población con una verdadera cuota de autonomía local que haga efectiva la equiparación administrativa de La Graciosa con las islas a las que ahora iguala en dignidad por esta vía estatutaria. Ahora bien, si este Estatuto de Autonomía, es decir, esta Ley de carácter orgánico aprobada por el Congreso de los Diputados, no permite ningún margen de actuación para llenar de contenido esta denominación, ¿̨hemos de comprender que en realidad esta nueva denominación es un mero simbolismo, vacío de instrumentos que permitan un verdadero ejercicio de la autonomía que la Constitución española reconoce a las islas en los archipiélagos balear y canario ex art. 141.4 CE? Veámoslo por partes, pues son muchas las cuestiones que se suscitan desde un punto de vista del Derecho administrativo en torno a este nuevo estatus.

Primero, desde el punto de vista simbólico, que a priori es el más palpable, podemos apreciar que se ha procedido a la reforma de distintos símbolos, como el himno de la comunidad autónoma, que, estando regulado en la Ley 20/2003, de 28 de abril, del Himno de Canarias, ve modificar su letra para la sustitución de los versos repartido en siete peñas alusivo a las islas que conforman el archipiélago por el de repartido en ocho peñas, según recoge el acuerdo el consejo de Gobierno de Canarias, de 21 de enero de 2019, que insta a la Consejería de Presidencia, Justicia e Igualdad a la tramitación, con carácter de urgencia, de una iniciativa legislativa dirigida a ejecutar dicha modificación. A esta modificación se le debería unir más pronto que tarde la modificación de la bandera oficial, y la del escudo, para dibujar en ellas la nueva isla que ahora reconoce el EAC y que debería estar representada en estos emblemas como todas las demás ínsulas del archipiélago.

No cabe duda de que esta renovación de símbolos es la traducción a la realidad del reconocimiento de La Graciosa como octava isla canaria, que empieza a ver sus frutos en el tablero institucional. Sin embargo, reconocemos que estas apreciaciones simbólicas merecen un análisis muy exhaustivo, que desbordan el Derecho administrativo, y que pasan a ser objeto de análisis de otras disciplinas como la ciencia política o la sociología, en lo que se refiriere al marcado simbolismo de esta nueva consideración territorial. Nos referimos en realidad a una de las connotaciones más destacables de la denominación de La Graciosa como octava isla. Y es que, por abordar el ejemplo, las relaciones comerciales, afectivas y sociales que han unido tradicionalmente a Canarias con Venezuela ya habían forjado la consideración del país caribeño como la octava isla canaria en una denominación reconocida a nivel social, político, cultural e institucional. Por ello, comprobamos que la tradicional consideración de las islas Canarias como una suma de siete territorios (al que se le ha unido por afecto Venezuela) requiere de una revisión que permita responder a 
la integración y dignificación de La Graciosa como isla, compatible con las implicaciones culturales que esta operación aritmética ha comportado históricamente.

Por otro lado, y regresando a aspectos más puramente territoriales, no se nos puede escapar el hecho de que la nueva enumeración funciona, intencionada o accidentalmente, como una manera de equiparar el número de islas en ambas provincias canarias, de manera que tanto Las Palmas como Santa Cruz de Tenerife extenderían su territorio sobre un número de ínsulas idéntico. De este modo, se mantendría vigente la idea de paridad e igualdad entre islas y provincias tan recurrente en determinados resurgimientos del pleito insular, y que con la proclamación del primer Estatuto en los años 80 se hiciera palpable precisamente con el reparto de la representatividad parlamentaria bajo el principio de triple paridad. Aunque ello, nuevamente, encontraría una interpretación más acertada en otros campos del conocimiento como el de la ciencia política, que debería responder de la traducción de este fenómeno de equiparación provincial en su campo habida cuenta del peculiar mapa canario, en el que en realidad han sido más frecuentes las ambiciones insularistas que las de carácter provincialista.

En otro orden de cosas, y para terminar de valorar la cuestión de saber si el nuevo Estatuto de Autonomía se limita a reconocer la existencia de una isla vacía de competencias contradiciendo el derecho constitucionalmente proclamado en el art. 141 de nuestra Carta Magna, podemos establecer una serie de afirmaciones. A nuestro juicio, el Derecho no puede permanecer mudo ante esta inactividad del ejercicio de la autonomía que La Graciosa parece asumir con su dignificación de isla, incluso si el art. 65.1 EAC se cura en salud al declarar que la entidad resultante de esta declaración «estará agregada administrativamente a Lanzarote». Primero, porque, de ser así, el propio Estatuto estaría reconociendo ya no el absurdo de proclamar una entidad vacía de contenido, sino haciendo una declaración expresa de inconstitucionalidad al proclamar el nacimiento jurídico de una isla que no es tal en Derecho. Segundo, porque el propio legislador autonómico estaría perdiendo la oportunidad histórica de responder a la necesidad de organización de las poblaciones existentes en La Graciosa desde un punto de vista jurídico acorde con los actuales tiempos de gobernanza y participación.

De este modo, es evidente que las regulaciones actuales en materia de régimen local imposibilitan la ejecución de una fórmula tradicional tendente a igualar la isla de La Graciosa al resto de islas con la constitución de su signo más reconocible, el Cabildo Insular, pero obliga en su contenido a encontrar y poner en marcha aquellos instrumentos que de acuerdo con los principios de eficiencia y eficacia den sentido a esta declaración de igualdad institucional con que el estatuyente ha querido colocar a La Graciosa con respecto a sus iguales, el resto de islas. El Derecho administrativo conoce estos instrumentos, y los conoce de la mano del desarrollo de las políticas públicas actuales que buscan la participación de los ciudadanos, con lo que la equiparación insular podría perfectamente estructurarse alrededor de un sistema de representación y participación más ventajoso para la isla de La Graciosa en el Parlamento regional (con la asignación de un escaño específico para la isla) y el establecimiento de alguna relación de comunicación más estrecha con el Gobierno canario que la haga más autónoma del Ayuntamiento de Teguise y de la 
isla de Lanzarote, de la que en realidad continuaría formando parte administrativa y políticamente. Esta relación con el Gobierno de Canarias podría plantearse de múltiples formas, siendo tal vez la menos gravosa la de establecimiento de un delegado que permitiera un canal de comunicación y participación directa entre los habitantes de La Graciosa y el Ejecutivo regional, de modo que se conseguiría dotar a la isla de una de las bondades de contar con Cabildo propio (como es el refuerzo de la relación institucional isla-comunidad autónoma) sin los perjuicios económicos y materiales de constituirlo. Estas fórmulas podrían desarrollarse al abrigo de una eventual reforma de la Ley de cabildos, de manera que pudiera flexibilizarse para la isla de La Graciosa la asignación de un ente equiparable al Cabildo al que se le eximiese de requisitos fundamentales de su constitución como son la existencia de municipios a los que coordinar, de manera que en realidad se le estaría otorgando a dicha isla un tipo de entidad cuya naturaleza se encontraría a medio camino entre el Ayuntamiento y el Cabildo, y tal consideración necesitaría de un estudio en profundidad de la normativa estatal y autonómica en materia de régimen local y más específicamente de la creación de una entidad ad hoc para la respuesta a esta variación organizativa en la planta territorial del archipiélago canario.

No obstante lo anterior, la lista de posibilidades para el encaje de un nuevo modelo de organización de La Graciosa es más extensa, sirviendo a meros efectos enunciativos la propuesta de estos instrumentos, que no han de entenderse como una lista numerus clausus, sino muy al contrario como una manera de contribuir al debate jurídico en que se base la estructura del modelo organizativo de La Graciosa. De este modo, el legislador autonómico estaría desarrollando un verdadero espacio de actividad política y organizativa a la nueva isla que reconoce el estatuyente, completando el contenido de su declaración con una verdadera respuesta jurídica que no se quede en una mera solución de símbolos, y que no sólo observe el ordenamiento concernido, sino que lo desarrolle y termine de darle coherencia con la aplicación de medidas de administración eficientes y modernas.

Asentado todo lo anterior, podemos afirmar que la proclamación de La Graciosa como isla, al abrigo del nuevo EAC, comporta una significación legal que no puede limitarse a una mera actualización de los símbolos institucionales de la comunidad autónoma canaria. Muy al contrario, tal y como proclama nuestra Constitución y desarrolla la legislación en materia de régimen local, la isla ha de configurarse administrativamente de una manera determinada. Con ello, y contextualizado el caso particular de La Graciosa, está claro que tanto la voluntad del estatuyente como las posibilidades legales operadas por las reformas de racionalización y sostenibilidad en el ámbito del sector público implican la necesidad de buscar una solución ad hoc para la dignificación de La Graciosa y para dar contenido a su proclamación como isla. Esta solución no puede ser una de las tradicionalmente escogidas, la constitución de Cabildo propio, al ser inviable económica y legalmente; incluso la creación de un ente a medida puede ser una idea complicada de materializar desde un punto de vista legal y una respuesta insuficiente para abordar la organización administrativa insular de La Graciosa. La solución, por tanto, debe encontrarse en la aplicación de técnicas de gobernanza modernas, y principalmente en las políticas de participación que permitan, primero, saber cuáles son las aspiraciones de autogo- 
bierno de los habitantes de La Graciosa, y segundo, ofrecer un encaje jurídicamente viable a esas aspiraciones con la puesta en marcha de medidas eficientes que doten de autonomía local a esta isla. Estas medidas que hemos propuesto, como ya hemos dicho, pueden ser muy variopintas, completadas, debatidas, pero deben valer para dar una respuesta jurídica a la proclamación que el nuevo Estatuto de Autonomía realiza sobre la isla de La Graciosa.

\section{CONCLUSIÓN}

A modo de conclusión, podemos decir que la proclamación de La Graciosa como isla, operada en el nuevo EAC, se fundamenta en la atención a una demanda tendente a la dignificación de las habitantes de Caleta de Sebo y Pedro Barba, lo que desde un punto de vista jurídico encontraría un excelente objeto de investigación constitucional a propósito de los derechos fundamentales reivindicables en esta demanda, así como en otros campos de conocimiento, a los que probablemente les interesen las consecuencias que comporta en su ámbito esta reconsideración territorial.

Ahora bien, desde el prisma administrativo son otros los debates de interés, enfocados en la traducción de esta nueva consideración insular a las posibilidades organizativas de La Graciosa, y cuyas actuales limitaciones legales obligarían a desarrollar soluciones específicas para esta realidad. Hemos propuesto, a modo meramente enunciativo, soluciones muy distintas: la primera, que reconocemos irrealizable, de crear un Cabildo en dicha isla; la segunda, más viable, crear un ente ad hoc que permita una gestión más autónoma de la isla, y que podría encontrarse a medio camino entre un Ayuntamiento y un Cabildo; y la tercera, la más factible, que implicaría la ejecución de un catálogo de políticas de representación y participación más ventajosas para La Graciosa.

De esta manera, existen herramientas que posibilitarían un encaje novedoso para La Graciosa de acuerdo con los principios de eficiencia que informan al sector público español, que merecen ser estudiadas, debatidas y completadas. Estas se podrían concretar, en el plano de la representación, en la asignación de un escaño en el Parlamento regional a favor de La Graciosa, la dotación de un delegado del Gobierno autonómico en la isla y la integración de un delegado de La Graciosa en el Cabildo Insular de Lanzarote; y en el plano de la participación, en un desarrollo un poco más sensible de las últimas técnicas de gobernanza para fortalecer la voz de los habitantes de la isla, incluso si se mantuviese su actual organización como entidad integrada en el municipio lanzaroteño de Teguise. 


\section{BIBLIOGRAFÍA}

\section{DOCTRINA}

Benítez de Lugo, J.M., El municipio y sus elementos esenciales, Madrid, 1986.

Betancort Reyes, F.J. (coord.), El Estatuto de Autonomía de Canarias, Ley Orgánica 1/2018, de 5 de noviembre, Editorial Iustel, Madrid, 2019.

Caamaño Dominguez, F., La autonomía de los entes locales en positivo, Fundación Democracia y Gobierno Local, Barcelona, 2003.

CRIADO, J., Nuevas tendencias en la gestión pública. Innovación abierta, gobernanza inteligente y tecnologias sociales en unas Administraciones Públicas colaborativas, INAP, Madrid, 2016.

García Morillo, J., La configuración constitucional de la autonomía local, Editorial Marcial Pons ediciones jurídicas y sociales, Madrid, 1998.

Palomar Olmeda (dir.), La Administración en tiempo de Crisis, Aranzadi, Pamplona, 2012.

Parejo Alfonso, L. y Vida Fernández, J. (coords.), Los retos del Estado y la Administración del siglo XXI. Tomo II. Tirant Lo Blanch, Valencia, 2017.

Ramió, C., La administración Pública del Futuro (Horizonte 2050). Instituciones, politica, mercado y sociedad de la innovación, Editorial Tecnos, Madrid, 2017.

Rodríguez-Arana Muñoz, J. y Sarmiento Acosta, M.J. (dirs.), Comentarios a la Ley 27/2013, de 27 de diciembre de racionalización y sostenibilidad de la Administración Local, Editorial Marcial Pons, Granada, 2014.

SÁnchez Saez, A., Autonomía Local y Descentralización, Tirant Lo Blanch, Valencia, 2008.

Sarmiento Acosta, M., Las Competencias de los Cabildos Insulares, Cabildo Insular de Gran Canaria, Las Palmas de Gran Canaria, 1992.

Sarmiento Acosta, M., «Posición institucional, competencias y financiación de los Cabildos Insulares en el estado autonómico». En XX Coloquio de Historia Canario-Americana, Cabildo Insular de Gran Canaria, Las Palmas de Gran Canaria, 2012.

Villar Rojas y Suay Rincón (dirs.), Derecho Local de Canarias, Editorial Iustel, Madrid, 2013.

\section{JURISPRUDENCIA}

STC 225/1998, de 25 de noviembre (BOE núm. 312, de 30 de diciembre de 1998).

\section{Noticia}

«El Senado reconoce a La Graciosa como la octava isla canaria habitada», La Vanguardia, 26 de junio de 2018. https://www.lavanguardia.com/politica/20180626/45418824885/el-senado-reconoce-a-la-graciosa-como-la-octava-isla-canaria-habitada.html. Consultado el 30 de septiembre de 2019.

\section{Estadística}

INE (Instituto Nacional de Estadística). Consultado el 30 de septiembre de 2019. Disponible en http:// www.ine.es/nomen2/index.do. 
